\title{
Note on Scalar Fields Non-Minimally Coupled to $(2+1)$-Gravity
}

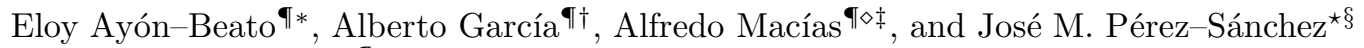 \\ - Departamento de Fúsica, CINVESTAV-IPN, \\ Apartado Postal 14-740, C.P. 07000, México, D.F., MEXICO. \\ $\diamond$ Departamento de Física, UAM-Iztapalapa, \\ Apartado Postal 55-534, C.P. 09340, México, D.F., MEXICO. \\ * Diploma Programme HEP, The Abdus Salam ICTP, \\ P.O. Box 586, 34100 Trieste, ITALY.
}

(October 31, 2018)

\begin{abstract}
Scalar fields non-minimally coupled to $(2+1)$-gravity, in the presence of cosmological constant term, are considered. Non-minimal couplings are described by the term $\zeta R \Psi^{2}$ in the Lagrangian. Within a class of static circularly symmetric space-times, it is shown that the only existing physically relevant solutions are the anti-de Sitter space-time for $\zeta=0$, and the Martínez-Zanelli black hole for $\zeta=1 / 8$. We obtain also two new solutions with non-trivial scalar field, for $\zeta=1 / 6$ and $\zeta=1 / 8$ respectively, nevertheless, the corresponding space-times can be reduced, via coordinate transformations, to the standard anti-de Sitter space.
\end{abstract}

file scalar.tex, 19.10.2000 .

PACS numbers: 04.50.+h, 04.60.Kz, 04.20.Jb, 04.70.Bw

Conformal solutions have been extensively studied in General Relativity, starting from most the general action coupled to scalar fields [1] 3 ]

$$
S=\frac{1}{2} \int d^{D} x \sqrt{-g}\left(\frac{1}{\kappa} C(\Psi) R-w(\Psi) \nabla_{\mu} \Psi \nabla^{\mu} \Psi+V(\Psi)\right)
$$

where $R$ is the Ricci scalar, $V(\Psi)$ is a potential function, $C(\Psi)$ and $w(\Psi)$ are coupling functions.

In four dimensions, the conformal solution discovered by Bekenstein [4], appears to be the only non-trivial black hole solution allowed for self-gravitating scalar fields non-minimally coupled to gravity. The study of the relevant system in $D>2+1$ dimensions has shown that some restrictions on the kind of plausible scalar field behaviors are required [5]. For example, in $(3+1)$-dimensions, the most general result including self-interactions and without cosmological constant, has been obtained by Mayo and Bekenstein [6.7]. In that work, spherical static non-trivial scalar field behaviors are excluded for non-minimal couplings with $\zeta<0$ and $\zeta \geq 1 / 2$. Consequently, since in $(3+1)$-dimensions the conformal coupling is $\zeta=1 / 6$ the Bekenstein black hole belongs to the non-covered range $(0<\zeta<1 / 2)$. In this sense, still remains open the question if in 4 -dimensions the value $\zeta=1 / 6$ is the unique coupling allowing a non-trivial scalar field behavior, or if there exist a family of solutions with non-minimally coupled scalar field behaviors within the interval under consideration.

Although the space-time is not three-dimensional, and $(2+1)$-dimensional gravity is clearly not a physically realistic model of our universe, it is a simple model which is rich enough to allow us to learn a good deal about the nature of quantum gravity. At first sight, the $(2+1)$-dimensional gravity looks trivial, in particular, the vacuum Einstein equations imply that space-time is locally flat, corresponding to the absence of the Weyl tensor in three dimensions. The triviality of local geometry in $(2+1)$-dimensional gravity holds even if the cosmological constant term is taken into account; the Einstein space is a space of constant curvature.

However, the black hole riddle has long been one of the most outstanding problems of modern physics. It has remained in focus for a long time as one of the potential testing grounds for quantum gravitational phenomena. As it is well known, three dimensional gravity, in vacuum, admits only the trivial locally flat $(2+1)$-Minkowski space. Thus, it is necessary either to couple matter to the theory, e.g., a cosmological constant or scalar matter, or to consider alternative vacuum or non-vacuum gravity theories in order to get solutions different from the trivial one. New solutions in $(2+1)$-gravity coupled to a self-interacting dilaton and in vacuum scalar-tensor theories have

\footnotetext{
${ }^{*}$ E-mail: ayon@fis.cinvestav.mx

${ }^{\dagger}$ E-mail: aagarcia@fis.cinvestav.mx

${ }^{\ddagger}$ E-mail: amac@xanum.uam.mx; macias@fis.cinvestav.mx

${ }^{\S}$ E-mail: iosephus@ictp.trieste.it
} 
been obtained by Chan [8]. Moreover, Chan and Mann [9] determined a conformal static black hole solution with a nontrivial conformal factor $\Psi$ for $C(\Psi)=1, w(\Psi)=4$, and $V(\Psi)=2 \Lambda \exp (b \Psi)$. A black hole solution with a negative cosmological constant coupled to a conformal scalar field for $C(\Psi)=1-\kappa(1 / 8) \Psi^{2}, w(\Psi)=1$, and $V(\Psi)=2 \Lambda / \kappa$ has been found by Martínez and Zanelli 10.

In this paper we consider a particular case of the action (1) in order to study non-minimal couplings of scalar fields to $(2+1)$-gravity with a cosmological constant term. Essentially, we work out a commonly used one-parametric family of theories with real parameter $\zeta$, where the non-minimal couplings are described by the term $\zeta R \Psi^{2}$ in the Lagrangian. We will show that for a static circularly symmetric metric, where the space-time possesses only one degree of freedom, the only existing non-trivial solutions are the anti-de Sitter space-time for $\zeta=0$, the MartínezZanelli black hole for $\zeta=1 / 8$. We obtain also two new solutions with non-trivial scalar field, for $\zeta=1 / 6$ and $\zeta=1 / 8$ respectively, whose space-time geometries reduce to the one of an anti-de Sitter space. In this way we are establishing, for the studied class, the completeness of the solutions to the corresponding field equations.

As mentioned above, in three dimensions our basic action reads

$$
\mathcal{S}=\frac{1}{2} \int d^{3} x \sqrt{-g}\left(\frac{1}{\kappa}\left[R+2 l^{-2}\right]-\nabla_{\mu} \Psi \nabla^{\mu} \Psi-\zeta R \Psi^{2}\right),
$$

where $\Lambda=-l^{-2}$ is the cosmological constant, $\Psi$ is the massless non-minimally coupled scalar field, and $R$ is the scalar curvature.

The field equations arising from (2) are, on one hand, the Einstein equations

$$
G_{\mu}{ }^{\nu}=l^{-2} \delta_{\mu}{ }^{\nu}+\kappa\left[\nabla_{\mu} \Psi \nabla^{\nu} \Psi-\frac{1}{2} \delta_{\mu}{ }^{\nu} \nabla_{\alpha} \Psi \nabla^{\alpha} \Psi+\zeta\left(\delta_{\mu}{ }^{\nu} \square \Psi^{2}-\nabla_{\mu} \nabla^{\nu} \Psi^{2}+G_{\mu}{ }^{\nu} \Psi^{2}\right)\right],
$$

and, on the other hand, the equation

$$
\square \Psi=\zeta R \Psi
$$

for the scalar field, where $\square$ is the Laplace-Beltrami operator.

We shall restrict our study to the following class of static circularly symmetric three-dimensional metrics, which, in polar coordinates, can be written as follows

$$
\boldsymbol{g}=-F(r) \boldsymbol{d} \boldsymbol{t}^{2}+F(r)^{-1} \boldsymbol{d} \boldsymbol{r}^{2}+r^{2} \boldsymbol{d} \boldsymbol{\theta}^{2}
$$

consequently, we assume that the scalar field only depends on the radial variable $r$, i.e., $\Psi=\Psi(r)$.

The Einstein equations (3), for the static circularly symmetric space-time (5), become

$$
\begin{gathered}
2 \zeta \Psi \Psi^{\prime \prime}+(2 \zeta-1)\left(\Psi^{\prime}\right)^{2}=0 \\
\left(1-\kappa \zeta \Psi^{2}-2 \kappa \zeta r \Psi \Psi^{\prime}\right) F^{\prime}-\kappa \Psi^{\prime}\left(4 \zeta \Psi+r \Psi^{\prime}\right) F=\frac{2 r}{l^{2}} \\
\left(1-\kappa \zeta \Psi^{2}\right) F^{\prime \prime}-4 \kappa \zeta \Psi \Psi^{\prime} F^{\prime}-\kappa\left(4 \zeta \Psi \Psi^{\prime \prime}+(4 \zeta-1)\left(\Psi^{\prime}\right)^{2}\right) F=\frac{2}{l^{2}} .
\end{gathered}
$$

where primes denote derivatives with respect to $r$. Eq. (6) corresponds to the combination $G_{r}{ }^{r}-G_{t}{ }^{t}$, and the Eqs. (7) and (8) are the components $G_{r}{ }^{r}$ and $G_{\theta}{ }^{\theta}$ of the Einstein equations (3), respectively. It is straightforward to show that the scalar field equation (4) follows from Eqs. (6)-(8) by using the Bianchi identity $\nabla_{\nu} G_{\mu}{ }^{\nu}=0$.

Let us proceed to integrate the field equations. For the general case, $\zeta \neq 0$, Eq. (6) becomes

$$
\left(\frac{\Psi^{\prime}}{\Psi}\right)^{\prime}-\frac{1-4 \zeta}{2 \zeta}\left(\frac{\Psi^{\prime}}{\Psi}\right)^{2}=0
$$

whose general solution can be immediately obtained, namely

$$
\Psi(r)=\frac{A}{(r+B)^{2 \zeta /(1-4 \zeta)}}
$$


where $A$ and $B$ are integration constants.

By substituting the expression (10) for $\Psi$ into Eq. (7), the following linear first order differential equation for the structural function $F$ is obtained

$$
\begin{aligned}
F^{\prime}= & \frac{\kappa \delta^{2} A^{2}((\delta-1) r-2 B)}{(r+B)\left(\kappa \delta A^{2}((\delta-1) r-B)+4(\delta+1)(r+B)^{\delta+1}\right)} F \\
& +\frac{8(\delta+1) r(r+B)^{\delta+1}}{l^{2}\left(\kappa \delta A^{2}((\delta-1) r-B)+4(\delta+1)(r+B)^{\delta+1}\right)}
\end{aligned}
$$

with $\delta \equiv 4 \zeta /(1-4 \zeta)$. It is straightforward to show that the general solution of the Eq. (11) is given by

$$
F(r)=\frac{4\left((\delta+1)\left(r^{2}-B^{2}\right)-l^{2} C\right)(r+B)^{\delta+1}}{l^{2}\left(\kappa \delta A^{2}((\delta-1) r-B)+4(\delta+1)(r+B)^{\delta+1}\right)},
$$

with $C$ a new integration constant.

It remains to fulfill Eq. (8), which via Eq. (6), becomes

$$
\left(1-\kappa \zeta \Psi^{2}\right) F^{\prime \prime}-4 \kappa \zeta \Psi \Psi^{\prime} F^{\prime}-\kappa\left(\Psi^{\prime}\right)^{2} F=\frac{2}{l^{2}} .
$$

It is easy to see that Eq. (13) imposes constraints on the integration constants of the form $A=A(B, \zeta)$ and $C=$ $C(B, \zeta)$. Moreover, by replacing in Eq. (13) the expressions (10) and (12) for $\Psi(r)$ and $F(r)$, and after some lengthy manipulations, the following algebraic equation is obtained:

$$
\begin{aligned}
8(\delta+1)^{3}[ & (\delta-1)(\delta-2) y^{4}-3 \delta(\delta-1) B y^{3}-\delta\left(l^{2} C-2(\delta+1) B^{2}\right) y^{2} \\
& \left.+(\delta+2) B l^{2} C y\right] y^{2 \delta} \\
-2 \kappa \delta(\delta+1) A^{2}[ & (\delta+4)(\delta+1)(\delta-1)^{2} y^{4}-\delta(\delta+1)\left(4 \delta^{2}+7 \delta-9\right) B y^{3} \\
& -\delta\left((\delta-1)^{2} l^{2} C-(\delta+1)(\delta+2)(5 \delta+1) B^{2}\right) y^{2} \\
& +(\delta+1) B\left(\left(2 \delta^{2}-3 \delta+4\right) l^{2} C-2 \delta(\delta+1)(\delta+2) B^{2}\right) y \\
& \left.-\delta^{2}(\delta+1) B^{2} l^{2} C\right] y^{\delta} \\
+\kappa^{2} \delta^{2} A^{4}[ & (\delta+1)(\delta-1)^{2} y^{4}-3 \delta(\delta+1)(\delta-1) B y^{3}+3 \delta^{2}(\delta+1) B^{2} y^{2} \\
& \left.-B\left((\delta-1) l^{2} C+\delta(\delta+1)(\delta+2) B^{2}\right) y\right]=0
\end{aligned}
$$

where $y$ stands for $y \equiv r+B$. Since the powers of $y$ are linearly independent functions [11], the corresponding coefficients must vanish independently. Therefore, different possibilities arise by assigning values or ranges of values to the parameter $\delta$ :

Let us first consider the case of positive values of $\delta$. For $\delta>0$, the highest power of $y$ in Eq. (14) is $2 \delta+4$, equating its coefficient to zero one gets the condition

$$
8(\delta+1)^{3}(\delta-1)(\delta-2)=0 .
$$

Hence, the possible solutions only exist for the values $\delta=1(\zeta=1 / 8)$ and $\delta=2(\zeta=1 / 6)$ of the coupling constant. For the first value, i.e., $\delta=1(\zeta=1 / 8)$, Eq. (14) becomes

$$
2\left(\kappa A^{2} B-4 C l^{2}+16 B^{2}\right)\left(8 y^{4}+3 \kappa A^{2} B y^{2}\right)-2 B\left(3 \kappa A^{2} B-4 C l^{2}\right)\left(24 y^{3}+B \kappa A^{2} y\right)=0,
$$

therefore, the vanishing of the coefficients of even and odd powers of $y$ yields the following relations 


$$
\kappa A^{2} B-4 C l^{2}+16 B^{2}=0=B\left(3 \kappa A^{2} B-4 C l^{2}\right) .
$$

Thus, in this case $(\delta=1, \zeta=1 / 8)$, there exist two admissible classes of solutions for the integration constants. The first class is obtained for

$$
A^{2}=\frac{8 B}{\kappa}, \quad C=\frac{6 B^{2}}{l^{2}},
$$

and it corresponds to the black hole of Martínez and Zanelli [10], i.e.,

$$
\Psi_{\mathrm{MZ}}=\sqrt{\frac{8 B}{\kappa(r+B)}}, \quad F_{\mathrm{MZ}}=\frac{(r+B)^{2}(r-2 B)}{r l^{2}},
$$

whose horizon is located at $r_{\mathrm{h}}=2 B$. In this case the free parameter $B$ is related to the mass $M$ of the hole through the relation $B=\sqrt{M l^{2} / 3}$.

The second class is achieved by setting $B=C=0$, and can be expressed as follows

$$
\Psi(r)=\frac{A}{\sqrt{r}}, \quad F(r)=\frac{r^{2}}{l^{2}} .
$$

This is a new conformal solution with a non-trivial scalar field behavior, although the corresponding space-time can be brought, via coordinate transformations, to the standard anti-de Sitter space form (see for example [12 [15]). Therefore, since the anti-de Sitter space-time is solution to the vacuum plus cosmological constant field equations, the energy-momentum tensor of the non-trivial scalar field is such that it vanishes identically (see the term in brackets on the left hand side of Eq. (3)). This peculiar behavior arises from the non-minimal coupling of the conformal scalar field to gravity.

For the second value, i.e., $\delta=2(\zeta=1 / 6)$. The highest power of $y$ in 14 is $y^{2 \delta+3}=y^{7}$, its coefficient vanishes if the constant $B$ is set equal to zero, then Eq. (14) reduces to

$$
-12\left(2 C l^{2}+\kappa A^{2}\right)\left(18 y^{6}-\kappa A^{2} y^{4}\right)=0 .
$$

Consequently

$$
C=-\frac{\kappa A^{2}}{2 l^{2}},
$$

and therefore,

$$
\Psi(r)=\frac{A}{r}, \quad F(r)=\frac{r^{2}}{l^{2}} .
$$

This is a new solution with a non-minimally coupled scalar field, however, it exhibits the same peculiarities as the ones of the previous solution Eq. (20).

In full, for positive values of the parameter $\delta$, we have established the existence of three classes of solutions, namely the Martínez-Zanelli black hole for $\zeta=1 / 8$, and two new solutions corresponding to the anti-de Sitter space with non-trivial scalar fields, one for $\zeta=1 / 6$, and one for $\zeta=1 / 8$.

Let us turn to the case of negative values of $\delta$. For $\delta<0$ the highest power of $y$ in Eq. (14) is $y^{4}$, the vanishing of its coefficient leads to the condition

$$
\kappa^{2} \delta^{2}(\delta+1)(\delta-1)^{2} A^{4}=0 .
$$

It should be noticed that $\delta \neq-1$, since the value $\delta=-1$ corresponds to infinite coupling constants $\zeta= \pm \infty$. Therefore, Eq. (24) implies that $A=0$ and leads to the condition

$$
8(\delta+1)^{3}\left[(\delta-1)\left[(\delta-2) y^{4}-3 \delta B y^{3}\right]-\delta\left[l^{2} C-2(\delta+1) B^{2}\right] y^{2}+(\delta+2) B l^{2} C y\right] y^{2 \delta}=0 .
$$

It is easy to see that the coefficient of $y^{4+2 \delta}$ never vanishes. Consequently, no solutions exist for $\delta<0$.

The remaining case corresponds to the minimal coupling $\delta=0$, in this case, Eq. (6) implies that $\Psi=$ const. and Eq. (7) integrates directly to 


$$
F(r)=\frac{r^{2}}{l^{2}}-M, \quad \Psi=\text { const. }
$$

This solution corresponds to the static BTZ solution [16], which is once again no other than the $(2+1)-$ anti-de Sitter space-time (see [12 15$]$ ).

It is important to stress the fact that the three solutions (19), (20), and (23) of the Einstein equations (6) -(8), also satisfy the scalar field equation (14).

To conclude this letter let us summarize the results.

We have established the complete class of solutions within the static circularly symmetric ansatz (5) for the structural function $F=F(r)$, and found that there only exist solutions for the following values of the non-minimal coupling constant $\zeta$ :

- For conformal coupling, $\zeta=1 / 8$, there exist two solutions, the first one corresponds to the conformal black hole (19) of Martínez and Zanelli, and the second one is a new conformal solution (20), whose space-time corresponds to the anti-de Sitter space, with a non-trivial scalar field.

- The non-minimal coupling $\zeta=1 / 6$ leads to a new solution (23), which space-time geometry is once again reducible to the anti-de Sitter form endowed with a non-trivial scalar field.

- For minimal coupling $\zeta=0$, the scalar field reduces to a constant, and the resulting space-time corresponds to the $(2+1)$-anti-de Sitter space-time (26).

The possibility of obtaining more general static, circularly symmetric solutions, with two structural functions is still open. The corresponding field equations are much more involved and hard to integrate in general. Nevertheless,

efforts have to be undertaken to overcome the difficulties in the integration process of this more general dynamical system.

\section{ACKNOWLEDGMENTS}

We thank Friedrich W. Hehl for useful discussions and literature hints. This research was partially supported by CONACyT Grants 32138E and 28339E, and by FOMES Grant: P/FOMES 98-35-15.

[1] P.G. Bergmann, Int. J. Theor. Phys. 1, 25 (1968).

[2] R. Wagoner, Phys. Rev. D1, 3209 (1970).

[3] T. Singh and L.N. Rai, Gen. Rel. Grav. 15, 875 (1983).

[4] J.D. Bekenstein, Ann. Phys. (NY) 82, 535 (1974).

[5] A. Saa, Phys. Rev. D53, 7377 (1996).

[6] A.E. Mayo, and J.D. Bekenstein, Phys. Rev. D54, 5059 (1996).

[7] J.D. Bekenstein, Proceedings of Second Sakharov Conference in Physics, Moscow, eds. I.M. Dremin, A.M. Semikhatov, 761 (World Scientific, Singapore 1997).

[8] K.C.K. Chan, Phys. Rev. D55, 3564 (1997).

[9] K.C.K. Chan and R.B. Mann, Phys. Rev. D50, 2600 (1994).

[10] C. Martínez and J. Zanelli, Phys. Rev. D54, 3830 (1996).

[11] L. Elsgoltz, Differential Equations and Variational Calculus (MIR, Moscow 1977).

[12] L.P. Einsenhart, Riemannian Geometry (Princenton Univ. Press, Princenton 1925).

[13] J. Plebański, Conformal Equivalent Riemannian Spaces (Monography CINVESTAV-IPN 1967).

[14] S. Weinberg, Gravitation 83 Cosmology (John Wiley \& Sons, New York 1972).

[15] A. García, "Anti-de Sitter Black-Hole-like Solutions and its Dimensional Reductions", preprint CINVESTAV-PHYSGRG-000501 (2000).

[16] M. Bañados, C. Teitelboim, and J. Zanelli, Phys. Rev. Lett., 69, 1849 (1992). 